RevMexAA (Serie de Conferencias), 20, 203-203 (2004)

\title{
THE RADIO : X-RAY CORRELATION IN CYGNUS X-3 AND OTHER GALACTIC MICROQUASARS
}

\author{
M. Choudhury, ${ }^{1}$ and A. R. Rao ${ }^{1}$
}

Cygnus X-3, a Galactic X-ray binary, shows the presence of outflows in the form of radio jets. The SED in the X-ray band shows a complicated structure and evolution. We review some recent results of the long-term correlation of the radio and $X$-ray emissions, chiefly in the low (hard) states of the source. Comparing the results with those of other Galactic microquasars, we attempt to provide a consistent picture of the accretion - ejection mechanism in these sources.

The X-ray binary Cygnus X-3 exhibits mainly two types of X-ray emissions, high (correspondingly soft) and low (correspondingly hard), although the individual spectral components are different compared to the canonical black hole states (Choudhury \& Rao 2002). During the low (hard) state, the soft X-ray emission (2-12 keV) is very strongly correlated to the radio emission $(2.2 \mathrm{GHz})$, whereas the hard X-ray $(20-100 \mathrm{keV})$ is anti-correlated to the both soft X-ray and radio (Choudhury et al. 2002). This is due to the pivoting of the X-ray spectral energy distribution (SED), correlated to the radio emission, with the pivot point lying between $10-$ $20 \mathrm{keV}$. Similar behaviour of the pivoting of the $\mathrm{X}$-ray SED with the radio emission is seen in the persistant black hole candidates GRS $1915+105$ and Cygnus X-1 (Choudhury et al. 2003), with the pivot point of GRS $1915+105$ lying near about $30 \mathrm{keV}$, while that of Cygnus X-1 lies in the region of 50 $100 \mathrm{keV}$ (Zdziarski et al. 2002). In the high (correspondingly soft) state, the radio emission shows a characteristic quenching of flux (not considering the flares) with the increase in X-ray flux (Figure 1). GX 339-4 also shows similar pivoting of the $X-$ ray spectra above $300 \mathrm{keV}$ (Wardzinski et al. 2002) in the low-hard state, correlated to radio emission, with quenched radio emission in the high-soft state (Corbel et al. 2000). The uniform behaviour of the various sources spanning 5 orders of magnitude of emitted flux (Figure 2) suggest the presence of uniform physical mechanism in this class of objects, viz. Two Component Accretion Flow (TCAF) model of

\footnotetext{
${ }^{1}$ Tata Institute of Fundamental Research, Mumbai-400005. India.(manojulu@tifr.res.in)
}

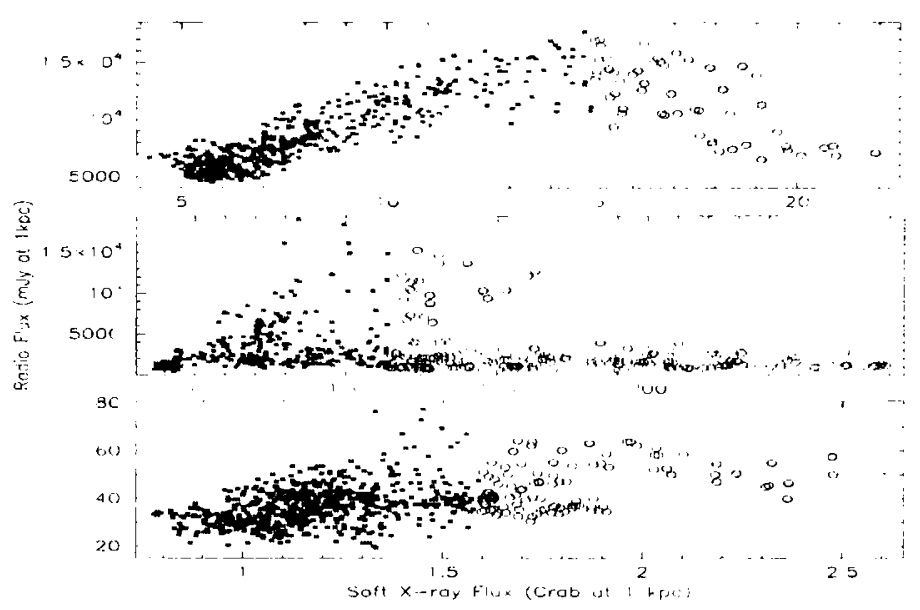

Fig. 1. Scatter diagram of soft $X$-ray emission and radio emission of Cygnus X-3 (top panel, GRS 1915+105 (middle panel) \& Cygnus X-1 (bottom panel), in the low as well as hard state, after removing the radio flares.

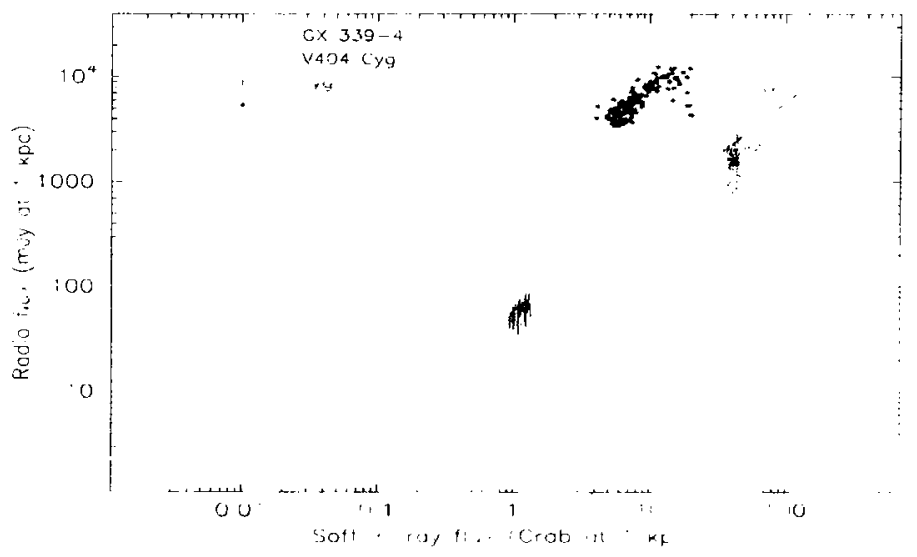

Fig. 2. Soft X-ray : radio correlation across various sources spanning 5 orders of magnitude of emitted flux.

Chakrabarti (1996). The Comptinising region is confined within the CENBOL, the extent of which, depending on the accretion rate, determines the X-ray SED evolution, while the radio emission is a function of the compression ratio, showing a turnover at the $\mathrm{X}$-ray spectral transition.

\section{REFERENCES}

Choudhury, M. et al. 2002, A\&A, 2002, 383, L35

Choudhury, M. et al. 2003, ApJ, 2003, 593, 452

Choudhury, M. \& Rao, A. R. 2002, JApA, 23, 39

Chakrabarti, S. K. 1996, Phys. Rep., 266, 229

Corbel, S. et al. 2000, A\&A, 359. 251

Wardzinski, G. et al. 2002, MNRAS. 337, 829

Zdziarski, A. A. et al. 2002, ApJ, 57 8,357 